\title{
340nm UV LED excitation in time-resolved fluorescence system for europium-based immunoassays detection
}

\author{
Rodenko, Olga; Fodgaard, Henrik; Tidemand-Lichtenberg, Peter; Pedersen, Christian
}

Published in:

Proceedings of SPIE

Link to article, DOI:

$10.1117 / 12.2250769$

Publication date:

2017

Document Version

Publisher's PDF, also known as Version of record

Link back to DTU Orbit

Citation (APA):

Rodenko, O., Fodgaard, H., Tidemand-Lichtenberg, P., \& Pedersen, C. (2017). 340nm UV LED excitation in time-resolved fluorescence system for europium-based immunoassays detection. In Proceedings of SPIE (Vol. 10072). [100720M] SPIE - International Society for Optical Engineering. Proceedings of SPIE - The International Society for Optical Engineering https://doi.org/10.1117/12.2250769

\section{General rights}

Copyright and moral rights for the publications made accessible in the public portal are retained by the authors and/or other copyright owners and it is a condition of accessing publications that users recognise and abide by the legal requirements associated with these rights.

- Users may download and print one copy of any publication from the public portal for the purpose of private study or research.

- You may not further distribute the material or use it for any profit-making activity or commercial gain

- You may freely distribute the URL identifying the publication in the public portal 


\title{
$340 \mathrm{~nm}$ UV LED excitation in time-resolved fluorescence system for europium-based immunoassays detection
}

\author{
Olga Rodenko ${ }^{* a, b}$, Henrik Fodgaard ${ }^{b}$, Peter Tidemand-Lichtenberg ${ }^{\mathrm{a}}$, Christian Pedersen ${ }^{\mathrm{a}}$ \\ ${ }^{a}$ Technical University of Denmark, Frederiksborgvej 399, 4000 Roskilde, Denmark; ${ }^{b}$ Radiometer \\ Medical ApS, Aakandevej 21, 2700 Broenshoej, Denmark
}

\begin{abstract}
In immunoassay analyzers for in-vitro diagnostics, Xenon flash lamps have been widely used as excitation light sources. Recent advancements in UV LED technology and its advantages over the flash lamps such as smaller footprint, better wall-plug efficiency, narrow emission spectrum, and no significant afterglow, have made them attractive light sources for gated detection systems. In this paper, we report on the implementation of a $340 \mathrm{~nm}$ UV LED based time-resolved fluorescence system based on europium chelate as a fluorescent marker. The system performance was tested with the immunoassay based on the cardiac marker, TnI. The same signal-to-noise ratio as for the flash lamp based system was obtained, operating the LED below specified maximum current. The background counts of the system and its main contributors were measured and analyzed. The background of the system of the LED based unit was improved by $39 \%$ compared to that of the Xenon flash lamp based unit, due to the LEDs narrower emission spectrum and longer pulse width. Key parameters of the LED system are discussed to further optimize the signal-to-noise ratio and signal-tobackground, and hence the sensitivity of the instrument.
\end{abstract}

Keywords: UV LED, time-resolved fluorescence, immunoassay, europium chelate, point-of-care

\section{INTRODUCTION}

Immunoassay instruments are employed in point-of-care $(\mathrm{PoC})$ testing performed near the patient (e.g., in emergency units and intensive care departments) in contrast to central laboratory ${ }^{1}$. One type of the instruments is fully automated heterogeneous analyzers. The available testing analytes include cardiac markers, hormones, inflammatory markers, thrombosis markers and others ${ }^{2}$.

Time-resolved fluorescence detection is a common technique in particles detection that allows suppressing fast decaying fluorescence background in nanosecond scale $^{3}$. The technique is usually employed using lanthanide chelates as fluorescence labels, due to their unique properties of large Stokes shift, long fluorescence lifetime up to a millisecond and narrow emission line $e^{4,5}$. The most common light sources for lanthanide chelates excitation were until recently Xenon flash lamps ${ }^{6,7}$. However, continuous advancements of UV LED technology make these solid state light sources highly competitive. The advantages include their availability at desired wavelengths, narrow spectral emission, higher efficiency, lower heat dissipation and easier control compared to flash lamps. $365 \mathrm{~nm}$ UV LEDs are widely used in e.g. flow cytometry ${ }^{8,9}$ and fluorescence microscopy ${ }^{10,11}$.

Until recently, $340 \mathrm{~nm}$ LEDs could provide only a few milliwatts and were only employed for some special applications ${ }^{12,13}$, but lower power levels prevented the employment in immunoassays. Now improvements of $340 \mathrm{~nm}$ UV LEDs have made them applicable with numerous lanthanide based chelates having excitation peaks in this wavelength range. In this manuscript, we report a case study of UV LED based excitation in immunoassays detection of cardiac marker Troponin I (TnI).

\section{EXPERIMENTAL SETUP}

The optical system is a reflection-type fluorimeter, see Figure 1. In the excitation light path, the light collected from the LED is imaged at the bottom of the test cup. Up to $80 \%$ of the light emitted from the LED is delivered to the bottom of the test cup. The image in the test cup is formed with a magnification of 5 , and measures $5 \times 5 \mathrm{~mm}^{2}$ in size. The

*olro@fotonik.dtu.dk

Optical Diagnostics and Sensing XVII: Toward Point-of-Care Diagnostics, edited by Gerard L. Coté, Proc. of SPIE Vol. 10072, 100720M - (C) 2017 SPIE · CCC code: 1605-7422/17/\$18 · doi: 10.1117/12.2250769 
fluorescence is collected by the emission optics and subsequently detected by a photomultiplier tube (PMT) operated in photon counting mode. The optical design as well as parameters of the LED were thoroughly discussed previously ${ }^{14}$.

A state-of-the art Xenon flash lamp optical unit (OU) was used as a reference light source. We have focused on differences between the two light sources before ${ }^{14}$. The sensitivity of the standalone flash lamp unit was adjusted before the experiment to a known standard. LED current of the LED based OU was adjusted to deliver the same energy per pulse as the flash lamp unit. Each test cup was measured consequently on the flash lamp unit and the LED unit without rotation (in the same cup holder).

Figure 1. Layout of the optical system.

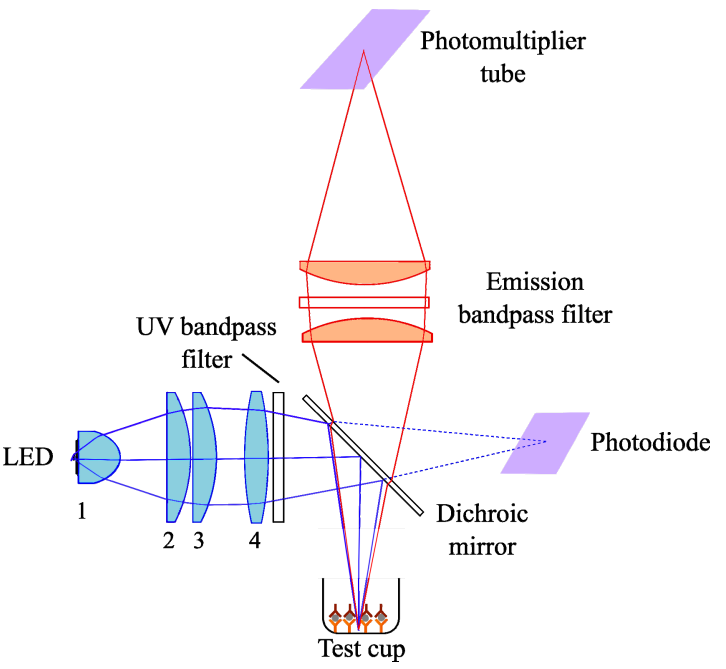

\section{SAMPLES PREPARATION}

The test cups were produced corresponding to all-in-one dry reagent technology ${ }^{15-17}$. The cups are made of UV absorbing polystyrene. Analyte-specific capture and tracer antibodies are placed in the bottom of the cup, see Figure 2. The tracer antibodies are marked with europium chelate label. The europium chelate synthesis and structure are described in a reference ${ }^{18}$. The capture and tracer antibodies, as well as an insulation layer which prevents contact between them during storage, are all in a ready-to-use dry format. Sample cups and blank cups were prepared for the experiment. The sample cup is processed with a $20 \mu \mathrm{L}$ reference solution with $\mathrm{TnI}$ at a concentration of $200 \mathrm{ng} / \mathrm{L}$ and assay buffer. During the incubation, it is heated to $37^{\circ} \mathrm{C}$ for 15 minutes and shaken to reduce the reaction time. After the reaction, the cup is washed and unbound tracer antibodies are washed out. The labeled tracer antibodies bound to the antigen are then excited by a UV light source in the measurement step. The fluorescence is then detected at $615 \mathrm{~nm}$. A blank cup is processed in the same way but with a solution of null TnI concentration. The counts measured on the blank cups are referred to as background counts.

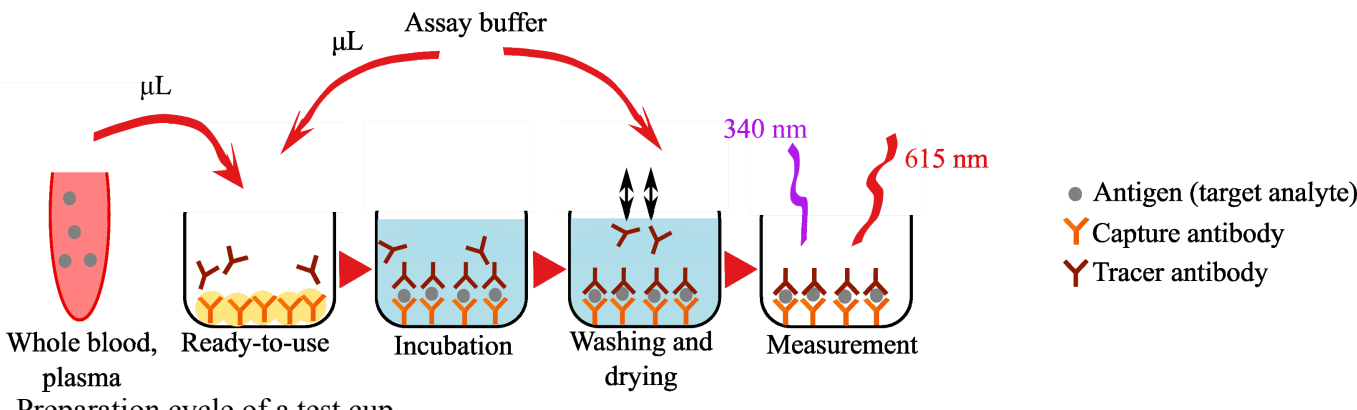

Figure 2. Preparation cycle of a test cup. 


\section{BACKGROUND CONTRIBUTIONS ANALYSIS}

We distinguish between the background contributors that are dependent on the optical system and excitation light and those which are independent from it. The background sources that depend on the optical system and the excitation light intensity include background from the OU itself (autofluorescence from materials and contamination) and from the test cup (polystyrene, streptavidin coating, non-specific binding, contamination). Moreover, we distinguish background from an empty cup (polystyrene only) and a blank cup (processed with null concentration solution). The latter contains chemical layer of antibodies in the bottom, as discussed above. These sources are independent from the useful fluorescence signal. The temperature-dependent PMT dark counts (less than 10 counts per second at $25^{\circ} \mathrm{C}$ ) are independent from the optical system and refer to the second group of the background contributors.

Correspondingly, we distinguish the following contributions to the noise in the measurements: shot noise of the useful fluorescence signal; shot noise of the background light (combining the intensity-dependent contributions) and the PMT dark noise. Other independent source is possibly the PMT amplifier circuit. The so far mentioned noise contributors refer to the measurement on one test cup. The cup-to-cup, lot-to-lot and instrument-to-instrument variations contribute to the noise as well. The noise contributions are independent from each other and therefore additive. The dominating noise source determines the signal-to-noise ratio of the measurements.
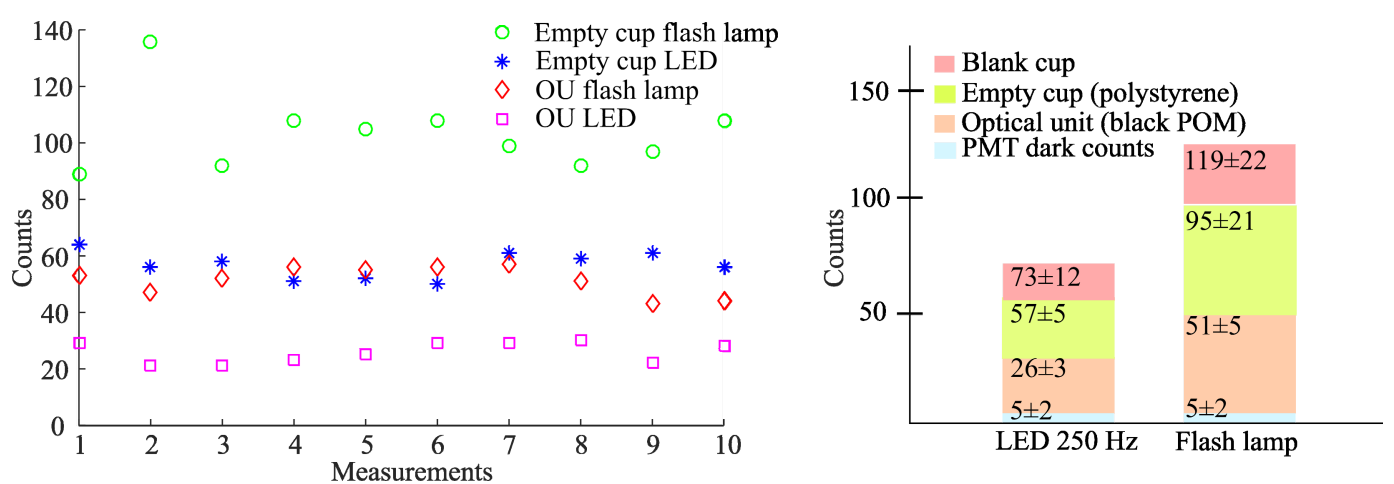

Figure 3. Background contributions of the LED- and flash lamp based systems, left: repetitive measurements of the OU itself, measured with the black POM closed volume fixture, and empty cups; right: differentiated background sources for the LED and flash lamp excitation: PMT dark counts, optical unit, empty cup (polystyrene only) and blank cup (processed cup with null concentration).

The background of the OU itself was measured with a light tight fixture made of black polyoxymethylene (POM). The fixture is a closed volume without test cup. All count values below are given for the total time of measurement (777 pulses at $250 \mathrm{~Hz}$ repetition frequency). For the LED based OU the background from the unit itself measured with the black POM was $26 \pm 3$ counts (including PMT dark counts), measured with an empty cup $57 \pm 5$ counts, and $73 \pm 12$ counts when measured on the blank cups, see Figure 3, right. The average values and standard deviations are calculated from the 10 repetitive measurements with the black POM and empty cups; and from the data of 15 replicates for the blank cups. The flash lamp based OU exhibits $51 \pm 5$ counts from the unit itself, $95 \pm 21$ including the empty cup and $119 \pm 22$ measured with the processed cup. The proportion of the four contributions is approximately the same for the two light sources but the total background level in the LED based unit is reduced by 39\%. The points in Figure 3, left are repetitive measurements performed on one black POM fixture (referred to as OU) and empty cups.

The background reduction is partially explained by the LEDs narrower emission spectrum comparing to the flash lamp and thus worse spectral match to the background excitation spectrum. Another reason is that the intensity of the longlived part of the background contributing to time gated measurements depends on the excitation pulse width. With longer pulse width, the number of molecules emitting unwanted light is lower and it drops faster if the background lifetime is shorter than the fluorophore lifetime. In addition, the amount of stray light is reduced in the LED based unit due to the optical system and absence of reflectors in the excitation light path, in contrast to the flash lamp. 


\section{TIME-RESOLVED DETECTION OF TNI PROTEIN IN IMMUNOASSAYS}

The performance of the UV LED based system was tested for time-resolved fluorescence measurements of TnI cardiac marker. Fifteen sample cups (with $200 \mathrm{ng} / \mathrm{L})$, and fifteen blank cups $(0 \mathrm{ng} / \mathrm{L})$ were produced for the experiment with $\mathrm{TnI}$ immunoassay.

Different definitions of SNR are used in literature. We define SNR as total detected signal corrected for averaged background value, divided by the sum of signal and background variations ${ }^{19}$

$$
S N R=\frac{\bar{S}-\bar{B}}{\sqrt{{\sigma_{S}}^{2}+{\sigma_{B}}^{2}}},
$$

where $\bar{S}$ is signal averaged over $\mathrm{N}$ sample cups, $\bar{B}$ is background averaged over $\mathrm{N}$ blank cups, $\sigma_{S}$ and $\sigma_{B}$ are standard deviations of the signal and background, respectively. $\bar{S}$ is the number of photons actually detected and $\bar{S}-\bar{B}$ is the useful fluorescence signal, or specific signal. Thus, in the denominator both signal and background variations are taken into account considering the propagation of uncertainty. Dark counts of PMT are neglected in this equation. In turn, we define signal-to-background ratio $(\mathrm{S} / \mathrm{B})$ as relation of averaged signal to average background $S / B=\frac{\bar{S}}{\bar{B}}$. Both SNR and S/B are calculated for a given concentration of analyte.

Sample cups with a concentration of cardiac marker TnI of $200 \mathrm{ng} / \mathrm{L}$ were measured with both optical units using the same excitation energy. Figure 4 presents the data for a total of 30 cups (15 sample and 15 blank cups). Table 1 shows parameters of the light sources as well as the statistical data. The samples were exposed to 777 pulses each with $5.1 \mu \mathrm{J}$ using a repetition frequency of $250 \mathrm{~Hz}$ corresponding to $1.27 \mathrm{~mW}$ average power. The average counts are listed with confidence intervals for a sample mean that is calculated from the t-parameter Student's distribution, corresponding to $95 \%$ confidence level. The sample standard deviations $\sigma$ are given with confidence intervals calculated from the $\chi^{2}$ distribution. For a confidence level of $95 \%$ and 15 samples, standard deviation confidence intervals are $[0.73 \cdot \sigma ; 1.58 \cdot \sigma]$. It should be noted that a sample size of 15 cups is not sufficient to have high statistical precision but it was not the goal in this investigation. It would require about 200 replicates to achieve a confidence interval for the standard deviation in the range, $[0.91 \cdot \sigma ; 1.08 \cdot \sigma]$ at a $95 \%$ confidence level. $\mathrm{CV}_{\mathrm{S}}$ and $\mathrm{CV}_{\mathrm{B}}$ are coefficients of variation for the cups with 200 $\mathrm{ng} / \mathrm{L}$ concentration and blank cups, respectively. These values are calculated using a relation between sample standard deviation and its mean value $\left(C V_{x}=\frac{\sigma_{x}}{\bar{x}}\right)$.

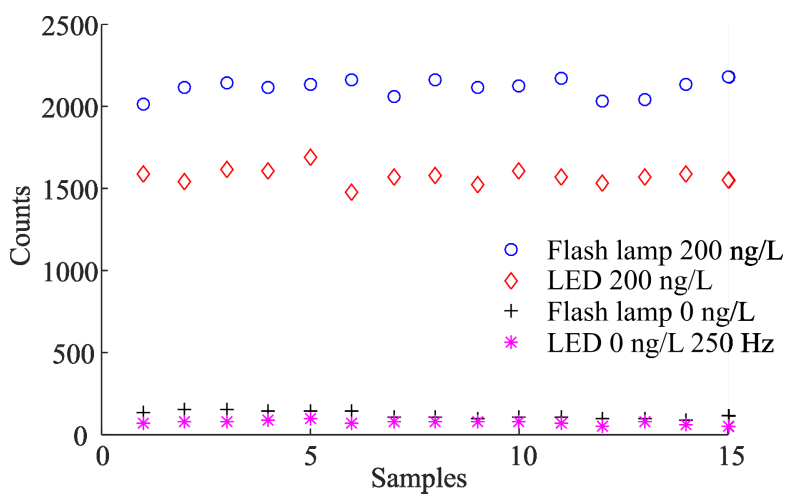

Figure 4. Total detected counts of 15 replicates with TnI $200 \mathrm{ng} / \mathrm{L}$ concentration and 15 blank cups processed with a solution of null concentration, excited by the flash lamp and LED based systems with equal excitation energy; each point represents one measurement of one test cup with 777 pulses. 
Firstly, the fluorescence response of sample cups is $26 \%$ lower when excited with the LED unit compared to the flash lamp excitation. We believe the main reason of the reduction is a less optimized overlap between the illumination area and the fluorescence emitting area. The UV LED illumination area in the test cup can be decreased at the cost of collected LED light. Another possibility is to change the size of the fluorescence emitting area in a test cup. It can be done for example by employing a spot coating ${ }^{16}$. In the spot coating approach, capture antibodies are pipetted in a very small volume in the test cup, thus determining the area of potential location of immunocomplexes. Combination of the two approaches (manipulating the excitation area and the fluorescence emitting area) allows maximization of the overlap. The factor of the area overlap appears to be dominating, as the other two factors of spectral and temporal parameters nearly cancel each other. The longer excitation pulse duration results in a signal reduction of $10 \%$, whereas the better spectral overlap of the LED emission is responsible for the $14 \%$ signal increase, compared to the flash lamp with equal pulse energy. Moreover, the information of the europium chelates distribution in the test cups is missing but could contribute to the signal change as well.

Secondly, the background level is reduced as discussed above. Overall the SNR is in the same range as for the flash lamp unit however decreased by $16 \%$ due to the lower signal level. The $\mathrm{S} / \mathrm{B}$ is improved only by $18 \%$ comparing to the background reduction of $39 \%$ for the same reason.

Table 1. Parameters of the excitation light sources and statistical data presented for sample concentration of $200 \mathrm{ng} / \mathrm{L}$ and blank cups (null concentration). Data calculated for 15 replicates.

\begin{tabular}{|c|c|c|c|c|}
\hline & \multicolumn{2}{|c|}{ Total signal (TnI $200 \mathrm{ng} / \mathrm{L})$} & \multicolumn{2}{|c|}{ Background (0 ng/L) } \\
\hline & Flash lamp & LED & Flash lamp & LED \\
\hline Average power, $\mathrm{mW}$ & & & 1.27 & \\
\hline Number of pulses & & & 777 & \\
\hline Frequency, Hz & & & 250 & \\
\hline Pulse energy, $\mu \mathrm{J}$ & & & 5.1 & \\
\hline Average counts $( \pm 95 \% \mathrm{CI})$ & $2114.1 \pm 28.6$ & $1574.3 \pm 26.8$ & $119.1 \pm 12.4$ & $73.3 \pm 7.1$ \\
\hline Sample standard deviation & 51.6 & 48.3 & 22.4 & 12.9 \\
\hline$[95 \% \mathrm{CI}]$ & {$[37.8 ; 81.4]$} & {$[35.4 ; 76.2]$} & {$[16.4 ; 35.3]$} & {$[9.4 ; 20.3]$} \\
\hline $\mathrm{CV}_{\mathrm{S}(\mathrm{B})}$ & $2.4 \%$ & $3.1 \%$ & $18.8 \%$ & $17.5 \%$ \\
\hline S/B@200 ng/L & 17.7 & 21.5 & & \\
\hline SNR@200 ng/L & 35.5 & 30.0 & & \\
\hline
\end{tabular}

There are several possibilities to improve the SNR of the LED based OU by optimizing different system parameters. As discussed above, decreasing the illumination area to the optimum value in the cup will result in higher fluorescence signal thus improved SNR. Decreasing the excitation pulse width will increase the signal however a maximum of $10 \%$ improvement can be reached ${ }^{14}$. Moreover, longer measurement window and shorter delay time are simple means of increasing the signal further.

The SNR grows with square root as a function of the excitation energy as Figure 5, left demonstrates this for a TnI concentration of $200 \mathrm{ng} / \mathrm{L}$ (fitted blue curve). The SNR was calculated for four replicates, shown as red circles. The values calculated from the experiment (with 15 replicates) are shown as blue square and magenta diamond for the flash lamp unit and the LED unit at $250 \mathrm{~Hz}$, respectively. The position of the data point for the flash lamp corresponds to the value of the LED current where the pulse energies of the flash lamp and LED units are equal. Higher statistical precision can be achieved increasing the number of samples. The S/B behavior depends mostly on the background contributor that dominates. The S/B ratio grows rapidly when excitation energy is small (lower current) and the signal is comparable to detection system noise, see Figure 5, right. The S/B reaches a constant value when the intensity-dependent unwanted fluorescence contributors, which grow with the excitation energy linearly, are considerably higher that the independent detection background sources. At this point $\mathrm{S} / \mathrm{B}$ reaches a plateau and is independent on the excitation energy as the dominating source being the fluorescence from the OU and the test cup. 

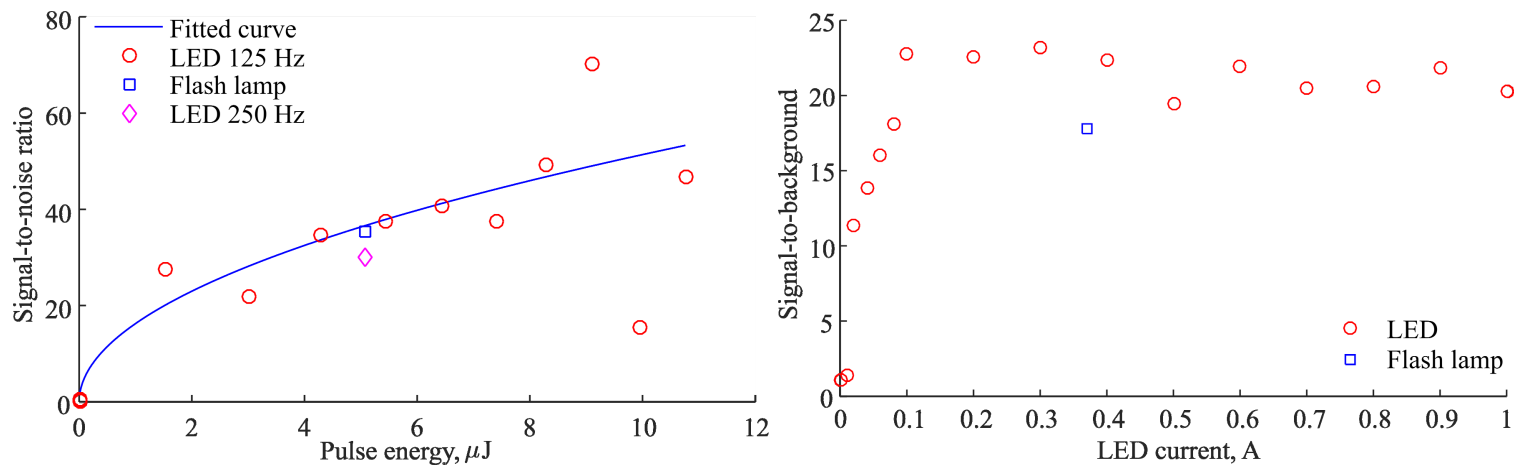

Figure 5. Left: SNR averaged for four cups vs. LED current (excitation pulse energy) for TnI concentration of $200 \mathrm{ng} / \mathrm{L}$; the SNR grows with square root with the excitation power; blue square and magenta diamond show mean values for the experiment with 15 replicates for the flash lamp and LED based unit, respectively; right: S/B ratio as a function of the LED current.

\section{CONCLUSION}

UV LED at $340 \mathrm{~nm}$ is successfully implemented in a time-resolved fluorescence system for immunoassay detection. Up to $80 \%$ of the UV LED excitation light is collected and imaged at the bottom of the test cup. The same SNR as for a state-of-the-art flash lamp unit was obtained with the designed UV LED based unit, without overdriving the LED.

The narrower emission spectrum of the LED when compared to the flash lamp, the longer pulse duration and its rapid switch-off time are important reasons for a 39\% decrease in the background fluorescence of the system. The S/B however decreased by only $18 \%$ because the fluorescence signal was decreased. Optimizing the overlap between the illumination area and fluorescence emitting area will allow for further improvement of the signal, the SNR and S/B.

\section{ACKNOWLEDGEMENTS}

The work was funded by Innovation Fund Denmark (grant 4135-00118B).

\section{REFERENCES}

[1] St-Louis, P., "Status of point-of-care testing: promise, realities, and possibilities," Clinical biochemistry 33(6), 427-440 (2000).

[2] Von Lode, P., "Point-of-care immunotesting: approaching the analytical performance of central laboratory methods," Clinical biochemistry 38(7), 591-606 (2005).

[3] Soini, E. and Kojola, H., "Time-resolved fluorometer for lanthanide chelates--a new generation of nonisotopic immunoassays," Clinical Chemistry 29(1), 65-68 (1983).

[4] Bünzli, J. C. G., "Lanthanide luminescence for biomedical analyses and imaging," Chemical reviews 110(5), 2729-2755 (2010).

[5] Hemmilä, I. and Laitala, V., "Progress in lanthanides as luminescent probes," Journal of Fluorescence 15(4), 529-542 (2005).

[6] Seveus, L., Väisälä, M., Syrjänen, S., Sandberg, M., Kuusisto, A., Harju, R., Salo, J., Hemmilä, I., Kojola, H. and Soini, E., "Time-resolved fluorescence imaging of europium chelate label in immunohistochemistry and in situ hybridization," Cytometry 13(4), 329-338 (1992).

[7] Connally, R., Veal, D. and Piper, J., "Flash lamp-excited time-resolved fluorescence microscope suppresses autofluorescence in water concentrates to deliver an 11-fold increase in signal-to-noise ratio," Journal of biomedical optics 9(4), 725-734 (2004).

[8] Kettlitz, S. W., Moosmann, C., Valouch, S. and Lemmer, U., "Sensitivity improvement in fluorescence-based particle detection," Cytometry Part A 85(9), 746-755 (2014).

[9] Jin, D., Connally, R. and Piper, J., "Practical time-gated luminescence flow cytometry. II: Experimental evaluation using UV LED excitation," Cytometry Part A 71(10), 797-808 (2007). 
[10]Connally, R., Jin, D. and Piper, J., "High intensity solid-state UV source for time-gated luminescence microscopy," Cytometry Part A 69(9), 1020-1027 (2006).

[11]Gahlaut, N. and Miller, L. W., "Time-resolved microscopy for imaging lanthanide luminescence in living cells," Cytometry Part A 77(12), 1113-1125 (2010).

[12]Peng, H., Makarona, E., He, Y., Song, Y. K., Nurmikko, A. V., Su, J., Ren, Z., Gherasimova, M., Jeon, S.-R., Cui, G. and Han, J., "Ultraviolet light-emitting diodes operating in the $340 \mathrm{~nm}$ wavelength range and application to time-resolved fluorescence spectroscopy," Applied Physics Letters 85(8), 1436-1438 (2004).

[13]Davitt, K., Song, Y. K., Patterson III, W. R., Nurmikko, A. V., Gherasimova, M., Han, J., Pan, Y.-L. and Chang, R., "290 and $340 \mathrm{~nm}$ UV LED arrays for fluorescence detection from single airborne particles," Optics Express 13(23), 9548-9555 (2005).

[14]Rodenko, O., Fodgaard, H., Tidemand-Lichtenberg, P., Petersen, P. and Pedersen, C., "340 nm pulsed UV LED system for europium-based time-resolved fluorescence detection of immunoassays," Optics Express 24(19), 22135-22143 (2016).

[15]Lövgren, T., Meriö, L., Mitrunen, K., Mäkinen, M. L., Mäkelä, M., Blomberg, K., Palenius, T. and Pettersson, K., "One-step all-in-one dry reagent immunoassays with fluorescent europium chelate label and time-resolved fluorimetry," Clinical chemistry 42(8), 1196-1201 (1996).

[16]Välimaa, L., Ylikotila, J., Kojola, H., Soukka, T., Takalo, H. and Pettersson, K., "Streptavidin-coated spot surfaces for sensitive immunoassays using fluorescence surface readout," Analytical and bioanalytical chemistry 391(6), 2135-2144 (2008).

[17]Pettersson, K., Katajamäki, T., Irjala, K., Leppanen, V., Majamaa-Voltti, K. and Laitinen, P., "Time-resolved fluorometry (TRF)-based immunoassay concept for rapid and quantitative determination of biochemical myocardial infarction markers from whole blood, serum and plasma," Luminescence 15(6), 399-407 (2000).

[18] Von Lode, P., Rosenberg, J., Pettersson, K. and Takalo, H., "A Europium Chelate for Quantitative Point-of-Care Immunoassays Using Direct Surface Measurement," Analytical Chemistry 75(13), 3193-3201 (2003).

[19] Seitzinger, N. K., Hughes, K. D. and Lytle, F. E., "Optimization of signal-to-noise ratios in time-filtered fluorescence detection," Analytical Chemistry 61(23), 2611-2615 (1989). 